\title{
OPTIMAL ADAPTIVE CONTROL OF FED-BATCH FERMENTATION PROCESSES
}

\author{
J.F. Van Impe* and G. Bastin** \\ *Katholieke Universiteit Leuven, Laboratory for Industrial Microbiology and Biochemistry, \\ B-3001 Heverlee, Belgium \\ **Université Catholique de Louvain, CESAME - Center for Systems Engineering and Applied Mechanics, \\ B-1348 Louvain-La-Neuve, Belgium
}

(Received June 1993; in final form March 1995)

\begin{abstract}
This paper presents a unifying methodology for optimization of biotechnological processes, namely optimal adaptive control, which combines the advantages of both the optimal control and the adaptive control approaches. As an example, the design of a substrate feeding rate controller for a class of biotechnological processes in stirred tank reactors characterized by a decoupling between biomass growth and product formation is considered. More specifically, the most common case is considered of a process with monotonic specific growth rate and non-monotonic specific production rate as functions of substrate concentration. The main contribution is to illustrate how the insight, obtained by preliminary optimal control studies, leads to the design of easy-to-implement adaptive controllers. The controllers derived in this way combine a nearly optimal performance with good robustness properties against modeling uncertainties and process disturbances. Since they can be considered model-independent, they may be very helpful also in solving the model discrimination problem, which often occurs during biotechnological process modeling. To illustrate the method and the results obtained, simulation results are given for the penicillin $G$ fed-batch fermentation process.
\end{abstract}

Key Words. Fed-batch fermentation processes; optimal control; adaptive control; linearizing control

\section{INTRODUCTION}

The design of high-performance model-based control algorithms for biotechnological processes is hampered by two major problems which call for adequate engineering solutions. First, the process kinetics are most often poorly understood nonlinear functions, while the corresponding parameters are in general time-varying. Second, up till now there has been a lack of reliable sensors suited to real-time monitoring of the process variables which are needed in advanced control algorithms. Therefore, the earliest attempts at control of a biotechnological process used no model at all. Successful state trajectories from previous runs which had been stored in the process computer were tracked using open-loop control. Many industrial fermentations are still operated using this method.

During the last two decades, two trends for the design of monitoring and control algorithms for fermentation processes have emerged (Bastin and Van Impe, 1995). In a first approach, the difficulties in obtaining an accurate mathematical process model are ignored. In numerous papers classical methods (e.g., Kalman filtering, optimal control theory, ...) are applied under the assumption that the model is perfectly known. Due to this oversimplification, it is very unlikely that a real- life implementation of such controllers -very often this implementation is already hampered by, e.g., monitoring problems- would result in the predicted simulation results. In a second approach, the aim is to design specific monitoring and control algorithms without the need for a complete knowledge of the process model, using concepts from, e.g., adaptive control and nonlinear linearizing control. A comprehensive treatment of these ideas can be found in the textbook by Bastin and Dochain (1990) and the references therein.

This paper shows how to combine the best of both trends into one unifying methodology for optimization of biotechnological processes: optimal adaptive control. This is motivated as follows. Model-based optimal control studies provide a theoretically realizable optimum. However, the real-life implementation will fail in the first place due to modeling uncertainties. On the other hand, model-independent adaptive controllers can be designed, but there is a priori no guarantee of the optimality of the results obtained. The gap between both approaches is bridged in two steps. First, heuristic control strategies are developed with nearly optimal performance under all conditions. These suboptimal controllers are based on biochemical knowledge concerning the process and on a careful mathematical analysis of the op- 
timal control solution. In a second step, implementation of these profiles in an adaptive, modelindependent way combines excellent robustness properties with nearly optimal performance. As an example, the design of a substrate feeding rate controller for a class of biotechnological processes in stirred tank reactors characterized by a decoupling between biomass growth and product formation is considered. More specifically, the most common case of a process with monotonic specific growth rate and non-monotonic specific production rate as functions of substrate concentration is investigated.

The paper is organized as follows. In Section 2 the concept of optimal adaptive control is motivated in detail. Starting from the optimal control solution, the heuristic substrate controllers described in (Van Impe et al., 1992; Van Impe, 1994) are briefly reconsidered with respect to a real-life implementation. It is indicated that a straightforward implementation is not robust at all. However, as these controllers are the translation of a realistic control objective, namely set-point control, they can serve as a basis for the development of more reliable, robust, model-independent adaptive control schemes. To do so, they are interpreted within the framework of nonlinear linearizing control theory. In this way, a mechanism is incorporated that makes them both stable and robust against disturbances.

The next step is then to cope with the monitoring problem, i.e., how to determine on-line the nonmeasurable variables needed in the controller, in other words, how to make the nonlinear linearizing controller adaptive. Sections 3, 4, and 5 present three possible solutions depending on which variables are on-line available. The use of software sensors is clearly illustrated. Both a linear regression estimator and a state-observer-based estimator can be used for on-line tracking of the unknown states and specific rates. According to the minimal modeling concept, these specific rates are considered as time-varying parameters. Furthermore, it is possible to take a measurement delay into account. The three solutions are compared with simulation results for the penicillin $G$ fedbatch fermentation process.

A remarkable result is as follows. If there are no state inequality constraints, a substrate concentration reference level is specified only for the second phase, namely the production phase. However, the proposed controllers can be implemented without difficulty from the start of the fermentation on. The first phase, the growth phase, is then mainly used to obtain estimator convergence. Furthermore, the extension to a problem with constraints is straightforward.
Finally, Section 6 presents some conclusions. The main contribution of this paper is to illustrate that the design of an adaptive controller for the considered class of fermentation processes can be successfully based on a preliminary optimal control study. For instance, a substrate concentration reference profile is designed which guarantees nearly optimal performance. As such, this paper can be considered as an extension of the methods presented in Chapter 5 of the textbook by Bastin and Dochain (1990), where the reference substrate concentration is kept constant throughout the whole fermentation process.

\section{OPTIMAL ADAPTIVE CONTROL: MOTIVATION}

\subsection{Problem statement}

This paper considers the class of fed-batch fermentation processes described by an (unstructured) model of the form:

$$
\begin{aligned}
& \frac{d S}{d t}=-\sigma X+C_{S, \text { in } u} \\
& \frac{d X}{d t}=\mu X \\
& \frac{d P}{d t}=\pi X-k_{h} P \\
& \frac{d V}{d t}=u
\end{aligned}
$$

where the state variables $S, X, P$, and $V$ are respectively the amount of the only limiting substrate in broth $[\mathrm{g}]$, the amount of cell mass in broth [g DW] (DW stands for dry weight), the amount of product in broth $[\mathrm{g}]$, and the volume of the liquid phase in the fermentor [L]. Dissolved oxygen is considered non-limiting, by maintaining a sufficiently high aeration level. The input $u$ of the system is the volumetric substrate feed rate $[\mathrm{L} / \mathrm{h}] . C_{S, \text { in }}$ (expressed in $[\mathrm{g} / \mathrm{L}]$ ) is the (constant) substrate concentration in the feed stream $u$, while $k_{h}[1 / \mathrm{h}]$ is the product hydrolysis or degradation constant. $\sigma, \mu$, and $\pi$ are respectively the (overall) specific substrate consumption rate $[\mathrm{g} / \mathrm{g} \mathrm{DW} \mathrm{h}]$, the (overall) specific growth rate $[1 / \mathrm{h}]$, and the specific production rate $[\mathrm{g} / \mathrm{g} \mathrm{DW}$ h]. These rates are interrelated by:

$$
\sigma=\mu / Y_{X / S}+m+\pi / Y_{P / S}
$$

with $Y_{X / S}$ the biomass on substrate yield coefficient $[\mathrm{g} \mathrm{DW} / \mathrm{g}], Y_{P / S}$ the product on substrate yield coefficient $[\mathrm{g} / \mathrm{g}]$, and $m$ the (overall) specific maintenance demand $[\mathrm{g} / \mathrm{g}$ DW $\mathrm{h}]$. When introducing the concept of endogenous fractions (Van Impe et al., 1992; Van Impe, 1994), all kinds of metabolism for biomass survival and product synthesis can be easily described within one unifying frame. The endogenous fraction $f_{m} \in[0,1]$ of 
the overall specific maintenance demand $m$, and the endogenous fraction $f_{P} \in[0,1]$ for product synthesis, are defined through the following equations:

$$
\begin{aligned}
& \mu \triangleq \mu_{\text {substr }}-Y_{X / S}\left(f_{m} m+f_{P} \frac{\pi}{Y_{P / S}}\right) \\
& \sigma \triangleq \frac{\mu_{\text {substr }}}{Y_{X / S}}+\left(1-f_{m}\right) m+\left(1-f_{P}\right) \frac{\pi}{Y_{P / S}}
\end{aligned}
$$

where $\mu_{s u b s t r}$ is the specific substrate-to-biomass conversion rate. Observe that equation (2) holds true independent of the value of the fractions $f_{m}$ and $f_{P}$. If $f_{m}=f_{P}=1$, then endogenous metabolism is assumed: biomass survival and product synthesis are assumed due to combustion of part of the biomass. On the other hand, if $f_{m}=f_{P}=0$, then maintenance metabolism is assumed: biomass survival and product synthesis are assumed due to consumption of part of the external substrate. If $\left.f_{m} \in\right] 0,1\left[\right.$ and $\left.f_{P} \in\right] 0,1[$, then a mixed maintenance/endogenous metabolism can be modeled.

If the concentrations $C_{S}, C_{X}$, and $C_{P}$ (defined as $S / V, X / V$, and $P / V^{-}$respectively) are used as state variables, the following equivalent model is obtained:

$$
\begin{aligned}
\frac{d C_{S}}{d t} & =-\sigma C_{X}-C_{S} \frac{u}{V}+C_{S, \text { in }} \frac{u}{V} \\
\frac{d C_{X}}{d t} & =\mu C_{X}-C_{X} \frac{u}{V} \\
\frac{d C_{P}}{d t} & =\pi C_{X}-C_{P} \frac{u}{V}-k_{h} C_{P} \\
\frac{d V}{d t} & =u
\end{aligned}
$$

In this paper, the shape of the specific rates $\mu_{s u b s t r}$ and $\pi$ as functions of substrate concentration $C_{S}$ is assumed as depicted in Fig. 1: $\mu_{\text {substr }}$ is a monotonically increasing function, while $\pi$ is non-monotonic exhibiting a maximum. In other words, the enzyme catalyzed production is not associated to the microbial growth. Observe that this model structure represents the most common case of fermentation processes with product formation and growth/production decoupling. Obviously, the specific rates $\mu_{\text {substr }}$ and $\pi$ may be functions of other component concentrations as well.

Consider the following optimization problem. Determine for the set of dynamic equations (1) [or (5)] the optimal volumetric substrate feed rate profile $u^{*}(t)$ which maximizes the final amount of product $P\left(t_{f}\right)$, subject to the following constraints:

1. $t_{0}=0, t_{f}=$ free

2. $P(0)=P_{0}$ and $X(0)=X_{0}$ are given. $S(0)=$
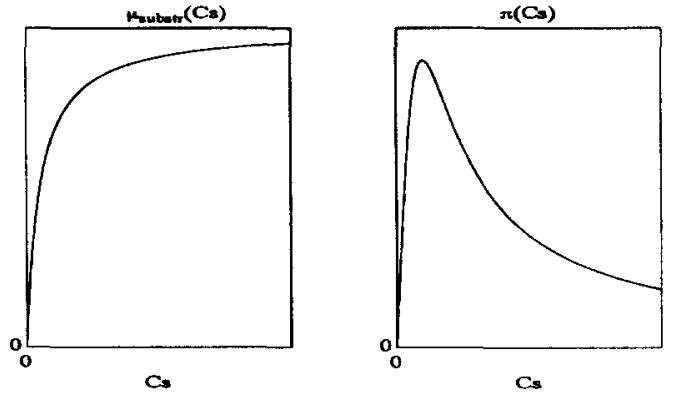

Fig. 1. Process with monotonic $\mu_{s u b s t r}$ and nonmonotonic $\pi$

$S_{0}$ is free. $V(0)=V_{0}$ follows from:

$$
V_{0}=V_{*}+S_{0} / C_{S, \text { in }}
$$

with $V_{*}$ the initial volume without substrate. Remember that substrate is added as a solution with concentration $C_{S, \text { in }}$.

3. The total amount of substrate available for fermentation, denoted by $\alpha$, is fixed. In other words, the final reactor volume is fixed to $V\left(t_{f}\right)=V_{f}$, with $V_{f}$ given

Observe that, from a mathematical point of view, the problem of determining both $S_{0}$ and $u^{*}(t)$ is equivalent to determining $u^{*}(t), t \in\left[0, t_{f}\right]$ with a Dirac input at time $t=0$.

\subsection{Case study: the penicillin $G$ fed-batch fer-} mentation process

As a test case for all methods presented in this paper, consider the penicillin $G$ fed-batch fermentation process modeled by Bajpai and Reuß (1980, 1981). This unstructured model is of the form (1) [or equivalently (5)], with the specific rates defined as follows:

$$
\begin{array}{rlrl}
\pi & =\pi_{m} \frac{C_{S}}{K_{P}+C_{S}+C_{S}^{2} / K_{I}} & \text { (Haldane) } \\
\mu_{\text {substr }} & =\mu_{C} \frac{C_{S}}{K_{X} C_{X}+C_{S}} & & \text { (Contois) }
\end{array}
$$

with $\pi_{m}$ the specific production constant [g/g DW h], $K_{P}$ the saturation constant for substrate limitation of product formation $[\mathrm{g} / \mathrm{L}], K_{I}$ the substrate inhibition constant for product formation, $\mu_{C}$ the maximum specific growth rate for Contois kinetics [1/h], and $K_{X}$ the Contois saturation constant for substrate limitation of biomass production $[\mathbf{g} / \mathbf{g} \mathrm{DW}]$. Observe that these specific rates have the general shape shown in Fig. 1.

Bajpai and Reuß assume a completely maintenance metabolism for both biomass survival and product synthesis. In other words, the endogenous fractions $f_{m}$ and $f_{P}$, as introduced in definitions (3) and (4), are both equal to zero: $f_{m}=f_{P}=0$. The extension to a mixed 
maintenance/endogenous or completely endogenous metabolism assumption has been described by Nicolaï et al. (1991) and Van Impe et al. (1992), with the endogenous fractions $f_{m}$ and $f_{P}$ modeled as functions of substrate concentration $C_{S}$.

In the application considered here, equations (3) and (4) reduce to:

$$
\begin{aligned}
& \mu=\mu_{s u b s t r} \\
& \sigma=\mu_{\text {substr }} / Y_{X / S}+m+\pi / Y_{P / S}
\end{aligned}
$$

which clearly satisfy relation (2).

Based on some experimental evidence, Bajpai and Reuß $(1980,1981)$ preferred Contois kinetics over the following, more commonly used Monod kinetics in modeling the specific substrate-to-biomass conversion rate $\mu_{\text {substr }}$ :

$$
\mu_{\text {stubstr }}=\mu_{M} \frac{C_{S}}{K_{S}+C_{S}} \quad \text { (Monod) }
$$

with $\mu_{M}$ the maximum specific growth rate for Monod kinetics $[1 / \mathrm{h}]$, and $K_{S}$ the Monod saturation constant for substrate limitation of biomass production $[\mathrm{g} / \mathrm{L}]$. One reason is that at high cell densities serious diffusional limitations can be expected which would cause the apparent value of the saturation constant $K_{S}$ in Monod kinetics to be higher than its value at lower cell densities. In Contois kinetics this behavior is modeled by the term $K_{X} C_{X}$ in the denominator. However, both kinetics have been shown to be valid for fungal growth. From the mathematical point of view, it is interesting to consider Monod kinetics as well, as all specific rates then become functions of substrate concentration $C_{S}$ only. A procedure to calculate the kinetic constants $\mu_{M}$ and $K_{S}$ from the Contois constants $\mu_{C}$ and $K_{X}$ can be found in (Van Impe, 1994). The numerical value of the original Bajpai and Reuss model parameters-i.e., involving Contois kinetics-is given in Table 2, together with the operational and initial conditions used in simulations. The value of $K_{P}$ and $K_{X}$ has been adjusted to obtain agreement with recent biochemical knowledge of the penicillin $\mathrm{G}$ fermentation (Van Impe, 1994). The results of a constant feeding strategy during $100 \mathrm{~h}$ are summarized in Table 1.

\begin{tabular}{|c|c|c|c|}
\hline \multicolumn{4}{|c|}{ parameters } \\
\hline$\mu_{C}$ & 0.11 & $\overline{K_{X}}$ & 0.06 \\
\hline$\pi_{m}$ & 0.004 & $k_{h}$ & 0.01 \\
\hline$K_{P}$ & 0.1 & $K_{I}$ & 0.1 \\
\hline$Y_{X / S}$ & 0.47 & $Y_{P / S}$ & 1.2 \\
\hline$m$ & 0.029 & $C_{S, \text { in }}$ & 500 \\
\hline \multicolumn{4}{|c|}{ initial conditions } \\
\hline$\overline{\overline{X_{0}}}$ & $\overline{10.5}$ & $\overline{\overline{S_{0}}}$ & to be specified \\
\hline$P_{0}$ & 0 & $V_{0}$ & $7+S_{0} / C_{S, \text { in }}$ \\
\hline$t_{0}$ & 0 & $\alpha$ & 1500 \\
\hline
\end{tabular}

Table 2 Parameters and initial conditions used in simulations

\subsection{Optimal control strategy}

The optimal control solution-in the sense of the Minimum Principle-for a general model (1) [or equivalently, (5)] has been analyzed by Van Impe (1994). Initial work along the same lines has been reported by Modak et al. (1986). Due to the decoupling between biomass growth and product synthesis, this type of fermentation behaves as a biphasic process.

The state vector $\mathbf{x}$ is defined as:

$$
\mathbf{x}^{\mathbf{T}} \triangleq\left[\begin{array}{llll}
S & X & P & V
\end{array}\right]
$$

while the control input $u$ is the volumetric substrate feeding rate. This paper is limited to a problem with an unconstrained state vector $\mathbf{x}$ and control input $u$, and free initial substrate concentration $C_{S}(0)$. The extension to problems involving one or more constraints is described in (Van Impe et al., 1993; Van Impe, 1994). The optimal substrate feed rate profile $u^{*}(t)$ can be characterized as follows (the results of the optimal control strategy for the penicillin $G$ fed-batch fermentation are summarized in Fig. 2 and Table 1):

1. The first phase, the growth phase, is a batch phase, i.e., $\left[u^{*}(t)=0,0<t<t_{2}\right]$ (see the upper plot of Fig. 2). All the substrate consumed during growth, denoted by $\alpha_{\text {growth }}$, is added all at once at time $t=0$, thus ensuring the highest possible specific growth rate $\mu$ for all $t \in\left[0, t_{2}\right]$, with a low production rate (see the lower plot of Fig. 2).

$\underline{\text { Table } 1}$ Constant control, optimal control, heuristic $C_{S}$-control, and optimal adaptive control results

\begin{tabular}{l|rccc}
\hline & $S(0)$ & $t_{2}$ & $t_{f}$ & $P\left(t_{f}\right)$ \\
\hline Constant control & 0 & - & 100.000 & 19.422 \\
Optimal control & 337 & 25.312 & 139.533 & 22.606 \\
Heuristic $C_{S}$-control with $C_{S}\left(t_{2}\right)=\left(K_{P} K_{I}\right)^{1 / 2}$ & 345 & 25.530 & 138.478 & 22.515 \\
Optimal adaptive control using $C_{S}$ and $C_{X}$ & 345 & - & 126.072 & 22.233 \\
Optimal adaptive control using $C_{S}$ & 345 & - & 137.538 & 22.484 \\
Optimal adaptive control using CER & 345 & - & 146.275 & 22.273 \\
\hline
\end{tabular}


2. During the second phase, the production phase, a singular control $\left[u_{s i n g}(t), t_{2}<t<\right.$ $t_{3}$ ] (see the upper plot of Fig. 2) forces the process to produce the product as fast as possible. At any time, there is a balance between glucose feeding and glucose demand for production and possibly maintenance, thus ensuring the lowest possible growth rate (see the lower plot of Fig. 2). When $V\left(t_{3}\right)=V_{f}$, the fermentation continues in batch $\left[u^{*}(t)=\right.$ $\left.0, t_{3}<t<t_{f}\right]$ until the net product formation rate $d P / d t$ equals zero at $t=t_{f}$.

This solution is similar to the one reported by San and Stephanopoulos (1989) who used $C_{S}$ as control input. In summary, the two-point boundaryvalue problem, which results from the application of the Minimum Principle and involves the state vector $\mathbf{x}$ and the so-called costate vector $\mathbf{p}$, has been reduced to the two-dimensional optimization of the initial substrate amount $S(0)=S_{0}$ [ or equivalently, $\left.C_{S}(0)\right]$, and the time $t_{2}$ at which the switching from batch to singular control occurs. It can be shown that for the performance measure considered here the singular control $u_{\text {sing }}(t)$ is a nonlinear feedback law of the state variables $S, X$, $P$, and $V$ only [see, e.g., (Modak et al., 1986; Van Impe, 1994)]. In other words, the costate vector p (which has no clear physical interpretation) has been eliminated completely.
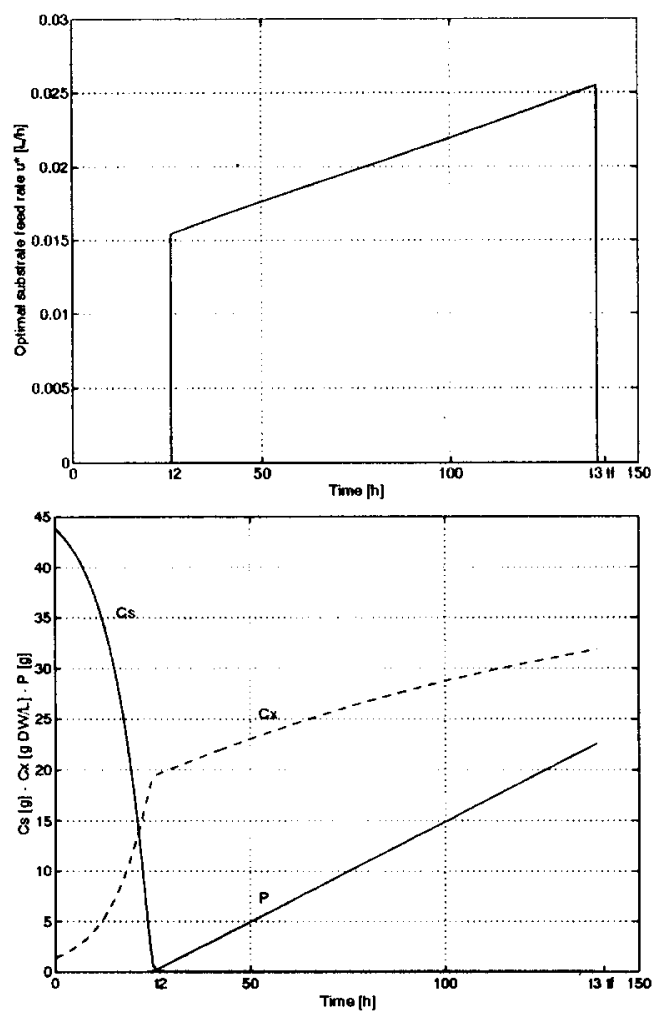

Fig. 2. Optimal control. Upper plot: optimal substrate feed rate profile $u^{*}(t)$. Lower plot: substrate concentration $C_{S}(t)$, biomass concentration $C_{X}(t)$, and product amount $P(t)$ profiles

\subsection{Heuristic control strategies}

The most important drawbacks of the optimal control solution can be summarized as follows.

1. Optimal control is a very model-sensitive technique. It requires a complete knowledge of the process model, including an analytic expression for all specific rates. Since in biotechnology this assumption is in practice never fulfilled, the optimal profile is generally calculated using a highly simplified model describing the process more or less correctly only from a qualitative viewpoint. Therefore, the resulting optimal profiles can be used only to increase the insight into both the process and the quality of the model.

2. For the performance measure considered in this paper [i.e., maximization of final product amount $\left.P\left(t_{f}\right)\right]$, the optimal feed rate profile is obtained in complete state feedback form except for the switching time $t_{2}$ between the batch growth phase and the singular production phase (Fig. 2). In general, $t_{2}$ must be determined numerically in advance.

3. Necessary and sufficient conditions can be derived for which $t_{2}$ also becomes a function of state variables only [see, e.g., (Van Impe, 1994)]. However, even if a perfect process model could be available which satisfies all conditions to obtain the complete optimal solution in closed loop, real-life implementation is still hampered by the lack of reliable sensors suited to real-time monitoring of the process variables needed in the controller. Besides a perfect analytical knowledge of all specific rates and corresponding parameters, the control during the singular phase $u_{s i n g}(t)$ requires on-line measurements of all state variables $S, X, P$, and $V$ (Van Impe, 1994).

Therefore, it is very useful to construct suboptimal strategies that do not suffer from the above difficulties, at the expense of as small as possible a decrease in performance. In (Van Impe et al., 1992; Van Impe, 1994) suboptimal heuristic controllers for both the substrate concentration $C_{S}$ (heuristic $C_{S}$-control) and the overall specific growth rate $\mu$ (heuristic $\mu$-control) are designed. As an example, in this paper heuristic $C_{S}$-control is considered, which can be motivated from both the microbiological and mathematical point of view.

2.4.1. Microbiological and experimental motivation. The construction of a suboptimal profile for the type of biotechnological processes under consideration can be based on the concept of a biphasic fermentation.

1. Growth phase $\left[0, t_{2}\right]$. During the growth phase the specific substrate-to-biomass conversion rate $\mu_{\text {substr }}$ is focused. For the con- 
trol needed reference is made to the optimal control results: in the case of an unbounded input $u$, an unconstrained state vector $\mathbf{x}$, and a free initial substrate concentration $C_{S}(0)$, the growth phase is a batch phase. In the case of a constraint on the input or the state and/or a fixed initial state, some minor modifications are required. A general strategy is that the fraction $\alpha_{\text {growth }}$ of the total amount of substrate available $\alpha$, which is consumed for biomass accumulation during the growth phase, must be added as fast as possible in order to obtain the highest possible value of Haubotr.

2. Production phase $\left[t_{2}, t_{3}\right]$. During production the specific production rate $\pi$ is focused. As shown in Fig. 1, $\pi$ exhibits a maximum as a function of the substrate concentration $C_{S}$. So, it is a reasonable control objective to keep the substrate concentration during the production phase constant at the level $C_{S, \pi}$ which maximizes $\pi$. For instance, in the case of Haldane kinetics, $C_{S, \pi}$ equals $\left(K_{P} K_{I}\right)^{1 / 2}$. Therefore, as soon as $C_{S}(t)$ equals $C_{S, \pi}$, the feed rate switches from $[u(t)=0]$ to:

$$
u_{\text {production }}=\frac{\sigma C_{X} V}{C_{S, \text { in }}-C_{S}}
$$

which keeps substrate concentration $C_{S}$ constant during production. This can be readily seen using model equations (5). Controller (6) is shut off when all substrate $\alpha$ has been added at time $t=t_{3}$, or equivalently, when $V\left(t_{3}\right)=V_{f}$. As in the case of optimal control, the fermentation continues in batch $\left[u(t)=0, t_{3}<t<t_{f}\right]$ until the net product formation rate $d P / d t$ equals zero at $t=t_{f}$.

Obviously, the switching time $t_{2}$ (Fig. 2) is known in closed loop: the production phase starts when the substrate concentration $C_{S}$ becomes equal to $C_{S, \pi}$. As a result, the optimization problem has been reduced to the one-dimensional optimization of the initial substrate concentration $C_{S}(0)$, or more generally, of the fraction $\alpha_{\text {growth }}$ of the total substrate amount available.

A further refinement of this strategy consists of optimizing the value of the substrate concentration level during production (denoted by $C_{S}^{*}$, which plays the role of a set-point). In other words, during production $C_{S}$ is kept constant, but not necessarily at the value $C_{S, \pi}$ which maximizes $\pi$. As in the case of optimal control, optimization of final product amount reduces to a twodimensional optimization problem. The degrees of freedom are the initial substrate concentration $C_{S}(0)$--or more generally, the fraction $\alpha_{\text {growth }}-$ and the substrate concentration set-point $C_{S}^{*} d u r-$ ing production.
2.4.2. Mathematical justification. The mathematical motivation of the proposed heuristic $C_{S^{-}}$ controller is based on the following considerations. If it is assumed that $\mu_{\text {substr }}$ and $\pi$ [and thus $\sigma$ through relation (2)] are functions of substrate concentration $C_{S}$ only-consider for instance the above model for penicillin fermentation with $\mu_{\text {substr }}$ modeled by Monod kinetics-then the optimal feed rate during the singular production phase $\left[t_{2}, t_{3}\right]$ is given by (Van Impe, 1994)

$$
\begin{aligned}
u_{\text {aing }}(t) & =\frac{\sigma C_{X} V}{C_{S, \text { in }}-C_{S}} \\
& +k_{h} \frac{p_{3} V\left(\pi^{\prime} X-\mu^{\prime} P\right)}{X\left(C_{S, \text { in }}-C_{S}\right)\left(p_{1} \sigma^{\prime \prime}-p_{2} \mu^{\prime \prime}-p_{3} \pi^{\prime \prime}\right)}(7)
\end{aligned}
$$

where a prime denotes derivation with respect to substrate concentration, and $p_{i}$ is the costate associated with component $x_{i}$ of the state vector $\mathbf{x}$. Note that this expression is linear in the specific product decay rate $k_{h}$, and a feedback law of state variables only (it can be shown that the costates $p_{1}$ and $p_{2}$ depend linearly on $p_{3}$ ). Furthermore, the second term requires knowledge of an analytical expression of the derivatives of all specific rates up to second order. It is shown in (Van Impe, 1994) that the proposed heuristic $C_{S}$-controller reduces to the optimal profile if (and only if) (i) the performance index is independent of final time $t_{f}$, (ii) the specific rates $\mu_{s u b s t r}$ and $\pi$ are functions of $C_{S}$ only, (iii) $k_{h}=0$, and (iv) the production phase starts when the substrate concentration $C_{S}$ reaches the level which maximizes the ratio $\pi / \sigma$. In cases where (some of) these conditions are not satisfied, the proposed heuristic $C_{S}$-controller is at least a very good approximation of the optimal solution.

Note that the specific rates $\mu_{\text {substr }}$ and $\pi$ can be allowed to be functions of other component concentrations as well (e.g., $C_{X}$ ), provided these specific rates as functions of substrate concentration $C_{S}$ have the general shape shown in Fig. 1. As an example, the results given in Table 1 are obtained for the penicillin fermentation process with $\mu_{\text {substr }}$ modeled by Contois kinetics.

\subsection{Linearizing control}

With respect to a real-life implementation, the heuristic controller (6) has the following advantages over the optimal controller (7). First, the switching time $t_{2}$ between growth and production (and thus the complete control) is known in closed-loop as a function of the state: $C_{S}\left(t_{2}\right)=$ $C_{S}^{*}$. Second, as for the modeling uncertainty problem, only the specific substrate consumption rate $\sigma$ is required. Third, as for the on-line monitoring problem, the number of state variables to be measured on-line has been reduced by one: there 
is no need for a measurement of the product $P$. This is an important advantage in cases where the product remains (almost) completely in the liquid phase of the reactor. Finally, the most important advantage is that the given optimal control problem-namely, optimization of the final product amount $P\left(t_{f}\right)$ at some unknown final time $t_{f}-$ has been replaced by a more common regulator problem-namely, regulation of substrate concentration $C_{S}$ to some set-point $C_{S}^{*}$ for all time $t$ during production-for which feedback control loops can be developed.

However, a real-life implementation is still far away. Two important problems remain to be solved.

Problem 1: The monitoring problem. Although the number of unknowns has been reduced, the heuristic $C_{S}$-controller still needs on-line measurements - or at least reliable estimates-of substrate $S$, biomass $X$, volume of the liquid phase $V$, and of the specific substrate consumption rate $\sigma$.

Problem 2: The stability problem. The closed-loop stability is not guaranteed a priori. From general model (5) the closedloop dynamics during production for substrate concentration $C_{S}$ when using controller (6) are simply:

$$
\frac{d C_{S}}{d t}=0
$$

Clearly, even a small disturbance can move substrate concentration irreversibly away from its desired value $C_{S}^{*}$, resulting in performance degradation.

In the following it is illustrated how to design controllers based on the heuristic approach that do not suffer from the above drawbacks.

\section{REMARK}

It is emphasized that the primary goal of a substrate feedback controller for a fed-batch fermentation process is not to stabilize the process globally, but rather to optimize it while keeping an inherently unstable type of behavior under control [see also (Bastin and Dochain, 1990)]. As an example, consider the growth phase of a fed-batch fermentation process with a substrate inequality constraint. The optimal strategy then consists of keeping $C_{S}$ at its maximum value, say $C_{S, M A X}$, using a control of the form (6) until all substrate available for growth $\alpha_{\text {growth }}$ has been added [see (Van Impe, 1994)]. The closed-loop dynamics of biomass are then, using (1) and (5):

$$
\frac{d X}{d t}=\mu\left(C_{S, M A X}\right) X
$$

$$
\begin{aligned}
\frac{d C_{X}}{d t} & =\left[\mu\left(C_{S, M A X}\right)\right. \\
& \left.-\frac{\sigma\left(C_{S, M A X}\right)}{C_{S, \text { in }}-C_{S, M A X}} C_{X}\right] C_{X}
\end{aligned}
$$

During growth, in general the specific growth rate $\mu$ is much larger than the dilution rate $D=u / V$. As a result, both the absolute amount $X$ and the concentration $C_{X}$ of biomass increase in an exponential way. Clearly, the substrate controller (6) does not stabilize the growth phase. It optimizes growth by keeping substrate concentration at its optimal value.

\subsection{The stability problem}

The second problem is considered first. When replacing the optimal controller (7) by the heuristic controller (6) the control objective becomes more realistic, namely set-point control or more generally tracking of a reference profile. The heuristic controller (6) performs well if there are no disturbances, measurement errors, ..., and if the switch from growth to production occurs exactly when $C_{S}\left(t_{2}\right)=C_{S}^{*}$. As in general these assumptions are not fulfilled, some mechanism must be incorporated in control law (6) which controls the tracking error in presence of disturbances, ... At this point the principle of linearizing control can be used. An introduction and several applications in bioreactor control can be found in (Bastin and Dochain, 1990) and the references therein.

1. In the application considered in this paper, the control variable is the volumetric feed rate $u$, while the controlled variable is the substrate concentration $C_{S}$. So an input/output model for this case is simply the first differential equation of $(5)$ :

$$
\frac{d C_{S}}{d t}=-\sigma C_{X}-C_{S} \frac{u}{V}+C_{S, i n} \frac{u}{V} .
$$

This input/output model (which is linear in the control $u$ ) is of relative degree one: the control $u$ appears explicitly in the first derivative (with respect to time $t$ ) of the controlled variable $C_{S}$.

2. A linear stable ( $\lambda$ is a strictly positive given number) reference model for the tracking error is then:

$$
\frac{d\left(C_{S}-C_{S}^{*}\right)}{d t}=-\lambda\left(C_{S}-C_{S}^{*}\right) .
$$

Note that the reference model is of the same degree as the input/output model. At this point, the reference signal $C_{S}^{*}$ may be timevarying.

3. A nonlinear linearizing controller is obtained 
by eliminating $d C_{S} / d t$ between (8) and (9):

$$
u_{0}=\frac{\sigma C_{X}+\frac{d C_{S}^{*}}{d t}-\lambda\left(C_{S}-C_{S}^{*}\right)}{C_{S, \text { in }}-C_{S}} V
$$

In the application considered here, a constant substrate concentration during the production phase is desired, so:

$$
u_{0}=\frac{\sigma C_{X}-\lambda\left(C_{S}-C_{S}^{*}\right)}{C_{S, \text { in }}-C_{S}} V
$$

In most practical situations the control action (i.e., the feeding pump capacity) is bounded. The resulting controller during the production phase is then:

$u_{\text {production }}=\left\{\begin{array}{lll}u_{0} & \text { if } & 0 \leq u_{0} \leq U_{M A X} \\ 0 & \text { if } & u_{0} \leq 0 \\ U_{M A X} & \text { if } & u_{0} \geq U_{M A X}\end{array}\right.$

\section{REMARKS}

1. Note that it is preferred to apply nonlinear linearizing control theory. Since a fermentation process is known to be inherently nonlinear, it is reasonable to expect that better control would result by exploiting from the outset the nonlinearities in the model in the design of a nonlinear adaptive control algorithm.

2. Obviously, this controller reduces to heuristic controller (6) if the tracking error $\left(C_{S}-C_{S}^{*}\right)$ due to disturbances, measurement errors, ..., equals zero.

3. An important advantage as compared with the heuristic controller (6) is the following. Controller (10) can be implemented from $t=0$ on, by considering $C_{S}^{*}$ as the set-point from $t=0$ on. This idea can be motivated as follows. If there are no inequality constraints on the state $\mathbf{x}$ and the initial substrate concentration is free, the control sequence is as shown in Fig. 2 with $u_{\text {sing }}$ replaced by the above nonlinear linearizing controller. The growth phase is a batch phase, as all substrate consumed during growth $\alpha_{\text {growth }}$ is added at time $t=0$ in order to maximize the specific growth rate $\mu$. In other words, there is no reference profile for substrate concentration during the growth phase. In general, the optimal initial substrate amount $S_{0}$ is sufficiently high so that during growth $C_{S}(t)>C_{S}^{*}$, with $C_{S}^{*}$ the desired substrate concentration level during production. As a result, the tracking error $\left(C_{S}(t)-C_{S}^{*}\right)$ is a very large positive number. Consequently, the control calculated using (10) is set equal to $u=0$, i.e., a batch phase as required. Furthermore, the feed rate switches automatically to positive values as soon as $C_{S} \rightarrow C_{S}^{*}$, so controller $(10)$ can indeed be implemented from $t=0$ on. As in the case of optimal and heuristic control, this is a twodimensional optimization problem. The degrees of freedom are the initial substrate concentration $C_{S}(0)$-or more generally, the fraction $\alpha_{\text {growth }}-$ and the reference substrate concentration during production $C_{S}^{*}$.

The extension to the case of a substrate inequality constraint $\left[C_{S}(t) \leq C_{S, M A X}\right]$ during growth is straightforward. The optimal initial substrate concentration is then (Van Impe, 1994) $C_{S}(0)=$ $C_{S, M A X}$. During growth, a controller of the form (10) tries to keep $C_{S}$ at $C_{S, M A X}\left[C_{S, M A X}\right.$ then plays the role of the reference value] until at some time instant $t=t_{1}$ the total amount of substrate reserved for growth, $\alpha_{\text {growth }}$, is added. At $t=t_{1}$ the reference level switches to the level $C_{S}^{*}$ which is optimal for production. Since at $t=t_{1}$ the actual substrate concentration $C_{S}$ is much larger than $C_{S}^{*}$, controller (10) switches to zero-i.e., batch mode-as required. If $C_{S} \rightarrow C_{S}^{*}$, controller (10) switches automatically to positive values to keep $C_{S}$ around $C_{S}^{*}$. Controller (10) can again be implemented from $t=0$ on. For this case the two degrees of freedom in the optimization are the time $t_{1}$-or equivalently, the amount of substrate reserved to growth $\alpha_{\text {growth }}$-and again the reference substrate concentration level during production $C_{S}^{*}$.

Both cases are illustrated for the penicillin $G$ fedbatch fermentation in Fig. 3 where it is assumed that the measurements are all perfect and that there are no disturbances. Observe that the nonlinear linearizing controller (10) becomes positive before $C_{S}$ reaches $C_{S}^{*}$. This guarantees a smooth transition in the substrate concentration profile at the start of the production phase. This behavior can be obtained using a small value of $\lambda$ (e.g., $\lambda=1$ ) in the reference model (9).

4. On the other hand, a large value of $\lambda$ increases the stability margin, tracking behavior and disturbance rejection. This can be easily seen when calculating the closed-loop response of substrate concentration $C_{S}$. Since $\lambda$ is at the disposal of the user, it can be used to search for an optimal trade-off.

\subsection{The monitoring problem}

In the following sections the first problem is considered, i.e., monitoring of all variables required in controller (10). Three solutions depending on which measurements are available on-line are presented. The remaining variables are then estimated on-line using software sensors. The three algorithms proposed are all based on the minimal modeling concept introduced in (Bastin and Dochain, 1990). In this approach no assumption 
is made concerning the exact analytical structure of the specific rates required in the control law, thus circumventing the modeling and corresponding parameter identification problem. Instead, they are treated as time-varying parameters which are estimated on-line. By doing so, the nonlinear linearizing controller (10) is made adaptive and can be implemented independently of the -usually unknown- analytical expression for the specific rates. Simulation results for the penicillin $G$ fedbatch fermentation process as described above illustrate each of the variants proposed.
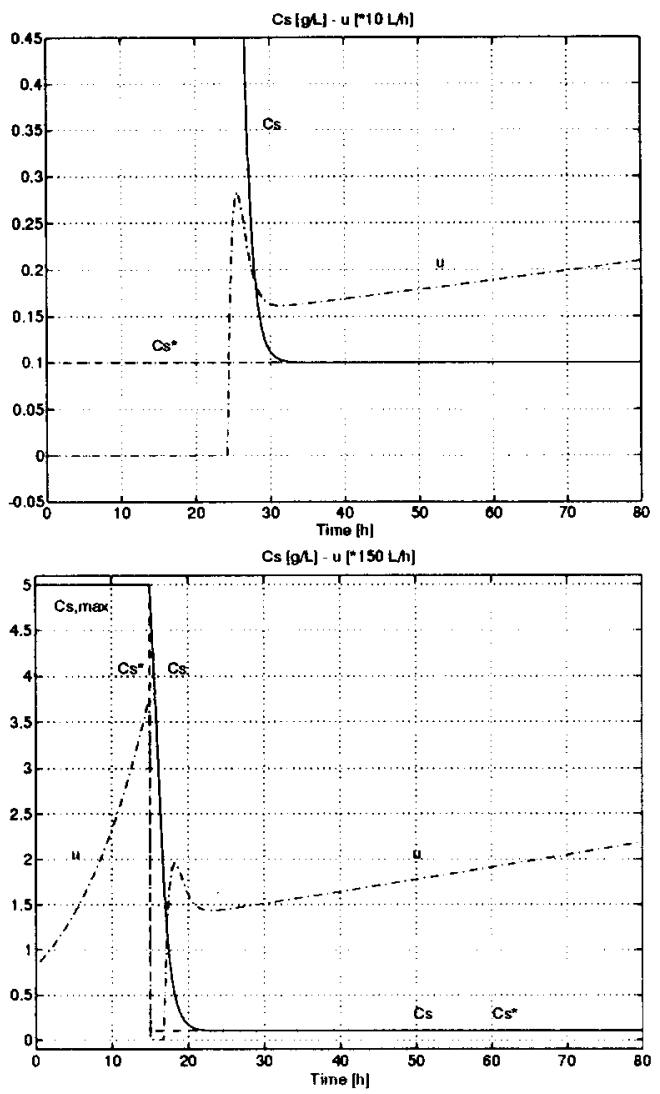

Fig. 3. Nonlinear linearizing control. Upper plot: no constraints. Lower plot: inequality constraint on $C_{S}$. Legend: - actual substrate concentration $C_{S},--$ reference profile $C_{S}^{*},-\cdot$ control action $u$

\section{OPTIMAL ADAPTIVE CONTROL: ON-LINE MEASUREMENTS OF $C_{S}$ AND $C_{X}$}

\subsection{Mathematical description}

The following assumptions are made.

1. Both substrate concentration $C_{S}$ and biomass concentration $C_{X}$ are measured on-line.

2. The results of the on-line measurement devices become available to the controller only after a time delay $\Delta T$, which is assumed equal for both measurements. This delay represents the time required to analyze a sample from the reactor contents.

3. The volume of the liquid phase $V$ is available on-line without time delay.

4. In agreement with the minimal modeling concept, no assumption is made concerning the exact analytical structure of the specific rates $\sigma$ and $\mu$.

In practice, a control algorithm will be implemented in discrete time. So the differential equations for $C_{S}, C_{X}$, and $V$ [see equations (5)] are discretized first. As an on-line measurement device can take a new sample from the reactor only after finishing the analysis of the previous one, the discretization interval is set equal to the sampling interval $\Delta T$. A first-order forward Euler discretization results in the following equations:

$$
\begin{aligned}
C_{S, k+1}-C_{S, k} & =-\sigma_{k} C_{X, k} \Delta T-C_{S, k} \frac{u_{k}}{V_{k}} \Delta T \\
& +C_{S, i n} \frac{u_{k}}{V_{k}} \Delta T \\
C_{X, k+1}-C_{X, k} & =\mu_{k} C_{X, k} \Delta T-C_{X, k} \frac{u_{k}}{V_{k}} \Delta T \\
V_{k+1}-V_{k} & =u_{k} \Delta T .
\end{aligned}
$$

The discrete-time version of linearizing controller (10) is:

$$
\begin{gathered}
u_{k}=\frac{\sigma_{k} C_{X, k}-\lambda\left(C_{S, k}-C_{S}^{*}\right)}{C_{S, \text { in }}-C_{S, k}} V_{k} \\
u_{\text {production }}= \begin{cases}u_{k} & , 0 \leq u_{k} \leq U_{M A X} \\
0 & , u_{k} \leq 0 \\
U_{M A X} & , u_{k} \geq U_{M A X}\end{cases}
\end{gathered}
$$

Besides the on-line measurement of $V_{k}$, controller (12) needs on-line estimates of $C_{S, k}, C_{X, k}$, and $\sigma_{k}$. Observe that $C_{S, i n}$ and $\lambda$ are prespecified constants.

Since the discrete-time model equations (11) are linear in the specific rates $\sigma$ and $\mu$, these rates can be estimated using a recursive least squares algorithm with forgetting factor $\zeta$ fed with on-line data of $C_{S}$ and $C_{X}$. A standard textbook formulation of RLS can be found in, e.g., (Goodwin and Sin, 1984).

However, due to the measurement delay, at time $t=k \Delta T$ only $C_{S, k-1}$ and $C_{X, k-1}$ are known. An adaptive version of controller (12) can then be obtained using the following algorithm (a hat " ${ }^{n \text { de- }}$ notes an estimate).

\section{Algorithm 1}

Step 1: Estimation of $\sigma_{k-1}$ and $\mu_{k-1}$ using $R L S$ Using equations (11) the residuals $\varepsilon$ can be written as: 


$$
\begin{aligned}
& \varepsilon_{C_{S, k-1}} \triangleq C_{S, k-1}-\hat{C}_{s, k-1} \\
& \quad=C_{S, k-1}+\hat{\sigma}_{k-2} C_{X, k-2} \Delta T \\
& \quad-\left(C_{S, i n}-C_{S, k-2}\right) \frac{u_{k-2}}{V_{k-2}} \Delta T-C_{S, k-2} \\
& \varepsilon_{C_{X}, k-1} \triangleq C_{X, k-1}-\hat{C}_{x, k-1} \\
& \quad=C_{X, k-1}-\hat{\mu}_{k-2} C_{X, k-2} \Delta T \\
& \quad+C_{X, k-2} \frac{u_{k-2}}{V_{k-2}} \Delta T-C_{X, k-2} .
\end{aligned}
$$

The gain $K$ is obtained via:

$$
\begin{aligned}
P_{k-1} & =P_{k-2} /\left(\zeta+C_{X, k-2}^{2} \Delta T^{2} P_{k-2}\right) \\
K_{k-1} & =-C_{X, k-2} \Delta T P_{k-1} .
\end{aligned}
$$

An estimation of $\sigma_{k-1}$ and $\mu_{k-1}$ is then:

$$
\begin{aligned}
& \hat{\sigma}_{k-1}=\hat{\sigma}_{k-2}+K_{k-1} \varepsilon_{C_{s, k-1}} \\
& \hat{\mu}_{k-1}=\hat{\mu}_{k-2}-K_{k-1} \varepsilon_{C_{X}, k-1} .
\end{aligned}
$$

Step 2: Prediction of $C_{S, k}$ and $C_{X, k}$ These variables can be calculated by using the estimates $\hat{\sigma}_{k-1}$ and $\hat{\mu}_{k-1}$ from Step 1 in the discrete equations (11) rewritten at time $k$.

Step 3: Calculation of the controller action $u_{k}$ $u_{k}$ is calculated by substituting the results of the previous steps in (12):

$$
u_{k}=\frac{\hat{\sigma}_{k-1} \hat{C}_{x, k}-\lambda\left(\hat{C}_{s, k}-C_{S}^{*}\right)}{C_{S, \text { in }}-\hat{C}_{s, k}} V_{k}
$$

In the above expression the required estimate $\hat{\sigma}_{k}$ is replaced by the (available) estimate $\hat{\sigma}_{k-1}$, as $\sigma$ varies only slowly as compared with the dynamics of the process.

\section{REMARKS}

1. Following a same line of reasoning as presented in Section 2.6 it can be concluded that this controller can also be implemented from $k=0$ on.

2. Instead of a linear regression estimator, an observer-based estimator could be used as well for the estimation of the specific rates $\sigma$ and $\mu$. The use of an observer-based estimator will be illustrated in the following sections.

3. Although the specific growth rate $\mu$ is not explicitly required in controller (12), an estimate is needed in order to predict the state at time $t=k \Delta T$ in Step 2 of the algorithm. Obviously, this complication is entirely due to the measurement delay $\Delta T$.

4. As already indicated in Section 2.6, the parameter $\lambda$ is at the disposal of the user to search for an optimal trade-off between smoothness of the controller action on the one hand, and stability margin and tracking behavior on the other.

\subsection{Simulation results}

Consider again the penicillin $\mathrm{G}$ fed-batch fermentation described in Section 2.2. All simulations are carried out using a continuous-time process model and a discrete-time controller action. Between two samples the controller action is kept constant.

In addition to the measurement time delay $\Delta T$, the on-line measurements of $C_{S}$ and $C_{X}$ are assumed to be corrupted by zero mean white noise. The standard deviation is set equal to $\operatorname{std}\left(C_{X}\right)=0.25 \mathrm{~g} / \mathrm{L}$, and $\operatorname{std}\left(C_{S}\right)=0.01 \mathrm{~g} / \mathrm{L}$. For a typical value of $C_{X}=10 \mathrm{~g} / \mathrm{L}$, this represents a standard deviation of $2.5 \%$. A typical value of substrate concentration $C_{S}$ during the production phase is the level $C_{S, \pi}$ which maximizes the specific production rate $\pi$. For the set of parameters given in Table $2, C_{S, \pi}$ is equal to $\left(K_{P} K_{I}\right)^{1 / 2}=0.1$ $\mathrm{g} / \mathrm{L}$. Under the above assumptions, a standard deviation on $C_{S}$ of even $10 \%$ is allowed.

For a measurement time delay $\Delta T=0.1 \mathrm{~h}$, the following results are obtained. The $R L S$ scheme is initialized with $P_{k=0}=10^{9}, \hat{\sigma}_{k=0}=0[\mathrm{~g} / \mathrm{g} \mathrm{DW}$ $\mathrm{h}]$, and $\hat{\mu}_{k=0}=0[1 / \mathrm{h}]$. The forgetting factor $\zeta$ is set equal to $\zeta=0.98$, while the parameter $\lambda$ is set equal to $\lambda=10$. The substrate concentration set-point during production $C_{S}^{*}$ is set equal to $C_{S}^{*}=C_{S, \pi}=0.1 \mathrm{~g} / \mathrm{L}$. When using the same initial substrate amount $S_{0}=345 \mathrm{~g}$ as given in Table 1 for the heuristic $C_{S}$-controller, a final product amount $P\left(t_{f}\right)=22.233 \mathrm{~g}$ is obtained at $t_{f}=126.072 \mathrm{~h}$, which comes very close to the optimal value $P_{\text {opt }}\left(t_{f}\right)=22.606 \mathrm{~g}$.

The convergence of the estimation of the specific rates $\sigma$ and $\mu$ is illustrated in the upper plot of Fig. 4. The lower plot shows the regulation of the actual substrate concentration $C_{S}(t)$ towards its set-point $C_{S}^{*}$, and the corresponding adaptive control action $u_{k}$. From both plots it can be concluded that the algorithm has converged shortly after the beginning of the production phase.

For $\Delta T=1 \mathrm{~h}$ the algorithm did not converge. Remember that $\Delta T$ represents both the sampling interval in discretizing the model equations and the measurement delay. As for Step 1 in the algorithm, it can be easily verified that there always exist an initial value $P_{k=0}$ and a forgetting factor $\zeta$ such that the estimations $\sigma_{k-1}$ and $\mu_{k-1}$ and the corresponding gain $K_{k-1}$ remain bounded if biomass concentration $C_{X}$ is strictly positive for all $t$ and if the control input $u$ is bounded. As a result, the instability at too large values of $\Delta T$ is completely due to the increasing inaccuracy of the predictions made in Step 2. It can be expectedand this has been confirmed during simulationsthat if the measurement delay $\Delta T$ becomes too 
large, especially the prediction of substrate concentration $C_{S}$ around the transition from growth to production becomes worse.

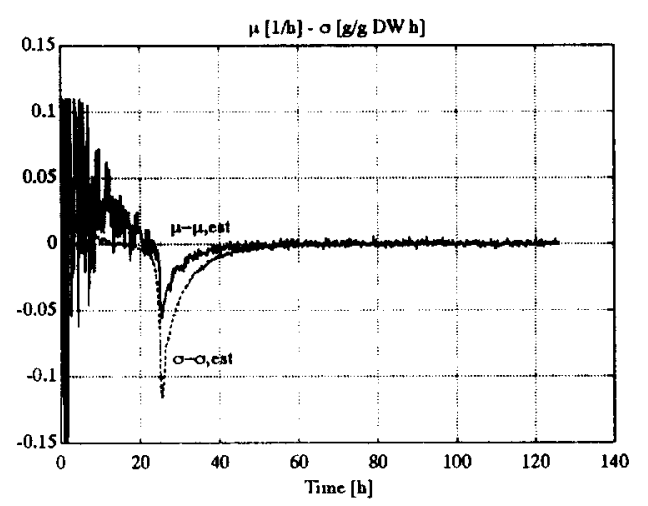

$\mathrm{Cs}[\mathrm{g} / \mathrm{L}] \cdot \mathrm{u}[\cdot 20 \mathrm{~L} / \mathrm{M}]$

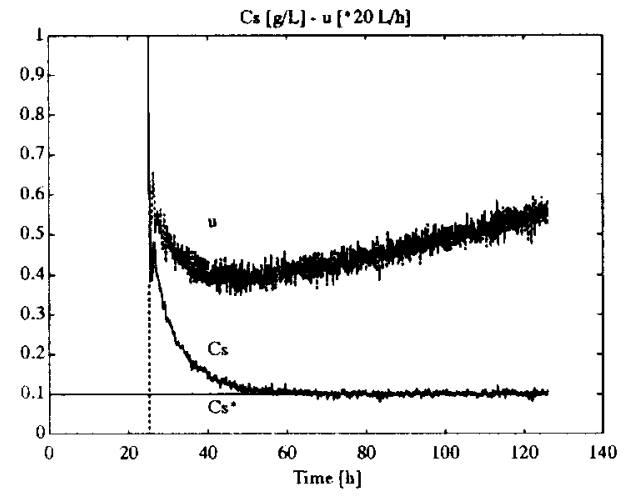

Fig. 4. Optimal adaptive control: on-line measurements of $C_{S}$ and $C_{X}$. Upper plot: estimation errors of $\sigma$ and $\mu$. Lower plot: regulation of $C_{S}$ and adaptive control action $u_{k}$

\section{OPTIMAL ADAPTIVE CONTROL: ON-LINE MEASUREMENTS OF $C_{S}$}

\subsection{Mathematical description}

The following assumptions are made.

1. Besides the volume $V$, substrate concentration $C_{S}$ is the only available on-line measurement.

2. In this and the next Section, a measurement time delay is not explicitly taken into account. If the measurement delay cannot be ignored, an additional prediction step must be incorporated in the discrete-time implementation of the proposed algorithms. An example is given in Section 3. Therefore, from now on $\Delta T$ only represents the discretization interval.

3. In agreement with the minimal modeling concept, no assumption is being made concerning the exact analytical structure of the specific rate $\sigma$.

An adaptive implementation of controller (10) can be obtained as follows. Since $C_{S, \text { in }}$ and $\lambda$ are known constants, only an on-line estimate of both the specific substrate consumption rate $\sigma$ and the biomass concentration $C_{X}$ is needed. More precisely, only an estimate of the product of $\sigma$ and $C_{X}$ is needed. Therefore, the rate $\beta$-with dimension $[\mathrm{g} / \mathrm{L} \mathrm{h}]-$ is defined as follows:

$$
\beta \triangleq \sigma C_{X}
$$

By doing so, the only unknown is exactly the rate $\beta$, which is considered as a time-varying parameter. In the following continuous-time algorithm, $\beta$ is estimated using a state-observer-based parameter estimator.

\section{Algorithm 2}

\section{Step 1: Estimation of $\beta$}

$$
\begin{aligned}
\frac{d \hat{C_{S}}}{d t} & =-\hat{\beta}+\left(C_{S, \text { in }}-C_{S}\right) \frac{u}{V}+\omega\left(C_{S}-\hat{C_{S}}\right) \\
\frac{d \hat{\beta}}{d t} & =-\gamma\left(C_{S}-\hat{C_{S}}\right)
\end{aligned}
$$

Step 2: Calculation of the controller action $u$

$$
\begin{aligned}
u_{0} & =\frac{\hat{\beta}-\lambda\left(C_{S}-C_{S}^{*}\right)}{C_{S, \text { in }}-C_{S}} V \\
u & = \begin{cases}u_{0} & \text { if } 0 \leq u_{0} \leq U_{M A X} \\
0 & \text { if } u_{0} \leq 0 \\
U_{M A X} & \text { if } u_{0} \geq U_{M A X}\end{cases}
\end{aligned}
$$

\section{REMARKS}

1. Tuning of the state-observer-based parameter estimator proposed in Step 1 reduces to the calibration of the (positive) constants $\omega$ and $\gamma$.

2. Just like the controller proposed in Section 3 , this controller does not need any a priori information either, such as, e.g., yield coefficients, ... Moreover, treating $\beta$ (and thus $\sigma$ ) as a timevarying parameter makes it robust against modeling uncertainties.

3. During simulations, a continuous-time process model and a discrete-time version of the above estimator and controller have been used. Between sampling instants -at distance $\Delta T$ - the controller action $u_{k}$ is kept constant. It can be easily shown that convergence of the estimator is guaranteed if the following inequalities are satisfied:

$$
\begin{aligned}
|1-\omega \Delta T| & \leq 1 \\
\left|1-\omega \Delta T+\gamma \Delta T^{2}\right| & \leq 1
\end{aligned}
$$

Observe that these constraints are independent of the design parameter $\lambda$ in the controller action $u_{k}$. 


\subsection{Simulation results}

Some simulation results for the penicillin $\mathrm{G}$ model presented in Section 2.2 are shown in Fig. 5. The sampling interval $\Delta T$ is set equal to $\Delta T=0.1$ h. The initial substrate amount $S_{0}$ is set equal to $S_{0}=345 \mathrm{~g}$, which is optimal for heuristic $C_{S^{-}}$ control (Table 1). Tuning of the estimator, initialized with $\hat{\beta}_{k=0}=0.25[\mathrm{~g} / \mathrm{L} \mathrm{h}]$, leads to the following efficient values: $\omega=1$, and $\gamma=10$.
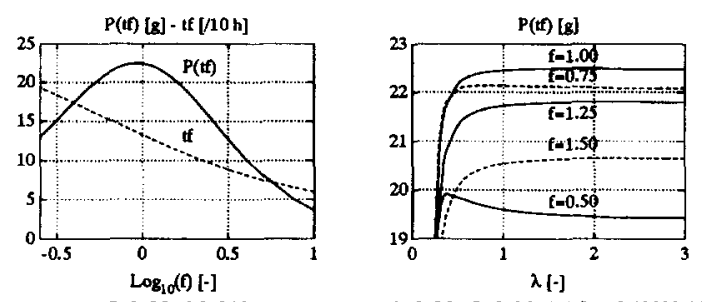

Cs $[g L] \cdot \beta[g /$
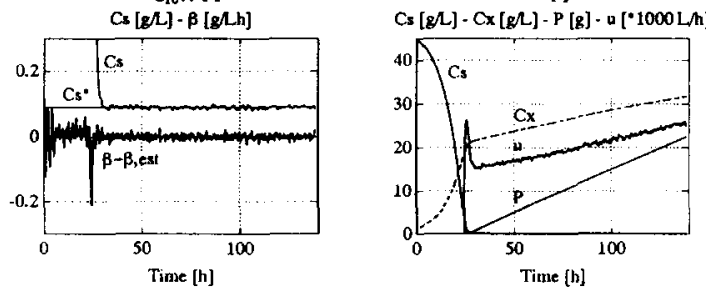

Fig. 5. Optimal adaptive control: on-line measurements of $C_{S}$. Upper left plot: optimization of $P\left(t_{f}\right)$ as function of $f$. Upper right plot: influence of $f$ and $\lambda$ on $P\left(t_{f}\right)$. Lower plots: time profiles for the set-point $C_{S}^{*}=0.9\left(K_{P} K_{I}\right)^{1 / 2}$ g/L

Define a factor $f$ as:

$$
f \triangleq \frac{C_{S}^{*}}{C_{S, \pi}} \equiv \frac{C_{S}^{*}}{\left(K_{P} K_{I}\right)^{1 / 2}}
$$

The results of an optimization with respect to this factor $f$-in other words, with respect to the setpoint during production-, and the controller parameter $\lambda$ are shown in the upper plots of Fig. 5. The left plots shows the final production $P\left(t_{f}\right)$ and the final time $t_{f}$ as functions of the factor $f$, for the controller parameter $\lambda$ equal to $\lambda=1$. Observe that the optimal value does not occur at $f=1$-in other words, at $C_{S}^{*}=C_{S, \pi^{-}}$, but at:

$$
C_{S, o p t}^{*}=0.9 C_{S, \pi}
$$

Due to the shape of the function $P\left(t_{f}\right)$ as function of $f$, it is clear that in practice three experiments should suffice to optimize the process. In addition, $f$ can be used to search for a trade-off between $P\left(t_{f}\right)$ and $t_{f}$. The upper right plot illustrates the influence of the controller parameter $\lambda$ on the final product amount $P\left(t_{f}\right)$, for different choices of the factor $f$. It can be seen that values of $\lambda$ larger than 1 have little influence upon the final product amount.
The lower plots show the time profiles for the optimal values $f=0.9$ and $\lambda=1$, while assuming a zero mean white noise on the measurements of $C_{S}$ with standard deviation $\operatorname{std}\left(C_{S}\right)=0.01 \mathrm{~g} / \mathrm{L}$. This represents an admissible standard deviation of $10 \%$ for substrate concentration within the order of magnitude $C_{S}=\mathcal{O}\left[\left(K_{P} K_{I}\right)^{1 / 2}\right]=\mathcal{O}[0.1]$ $\mathrm{g} / \mathrm{L}$ during production. The lower left plot illustrates the convergence of the estimator for the rate $\beta$, and the regulation of the actual substrate concentration $C_{S}(t)$ towards its set-point $C_{S}^{*}=0.09$ $\mathrm{g} / \mathrm{L}$. As in the previous Section, the proposed algorithm has converged shortly after the beginning of the production phase. The right plot shows the adaptive control action $u_{k}$, together with the corresponding profiles for the state variables. The final product amount is $P\left(t_{f}\right)=22.484 \mathrm{~g}$ at $t_{f}=137.538 \mathrm{~h}$, which comes again very close to the optimal value of $P_{\text {opt }}\left(t_{f}\right)=22.606 \mathrm{~g}$ (Table 1).

\section{OPTIMAL ADAPTIVE CONTROL: ON-LINE MEASUREMENTS OF CER}

\subsection{Mathematical description}

In the algorithms of Sections 3 and 4 , the major bottle-neck is the accuracy of the on-line substrate concentration measurements. Kleman et al. (1991) reported a control algorithm maintaining $C_{S}$ as tight as $0.49 \pm 0.04 \mathrm{~g} / \mathrm{L}$ during growth of $E$. coli. Using the parameter values of Table 2 , the optimal value for substrate concentration during production is in the order of magnitude $C_{S}=\mathcal{O}\left[\left(K_{P} K_{I}\right)^{1 / 2}\right]=\mathcal{O}[0.1] \mathrm{g} / \mathrm{L}$. Although the proposed algorithms proved robust against standard deviations of even $10 \%$, the question arises whether such a small, locally determined concentration level can be considered as representative for the whole reactor contents, which is in practice not perfectly mixed.

This problem can be circumvented as follows. In order not to overload the notation, a completely maintenance metabolism is assumed, i.e., $f_{m}=$ $f_{P} \equiv 0$ in expressions (3) and (4). In other words, the specific substrate-to-biomass conversion rate $\mu_{\text {substr }}$ is identical to the specific growth rate $\mu$. If the specific growth rate $\mu$ is a monotonically increasing function of substrate concentration $C_{S}$ (see Fig. 1), then prespecifying a reference profile for $C_{S}$ can be replaced by prespecifying a reference profile for the specific growth rate. In the case of $\mu$ function of $C_{S}$ only this is even identical. An appropriate reference profile is then: during growth $\mu$ should be as high as possible, while during production $\mu$ should be kept constant at $\mu=\mu^{*}$.

Obviously, an on-line estimation of the specific growth rate $\mu$ is required. This can be done, e.g., 
using the easily accessible measurement of $\mathrm{CO}_{2}$ in the effluent gas from the fermentor. The dissolved carbon dioxide dynamics are given by:

$$
\frac{d C_{C}}{d t}=C E R-D C_{C}-Q_{o u t}
$$

with $C_{C}$ the dissolved carbon dioxide concentration $\left[\mathrm{L} \mathrm{CO}_{2} / \mathrm{L}\right]$, and $Q_{\text {out }}$ the rate of outflow of carbon dioxide from the reactor in gaseous form [ $\mathrm{L}$ $\mathrm{CO}_{2} / \mathrm{L} \mathrm{h}$. This model is valid only if the dissolved carbon dioxide concentration $C_{C}$ is lower than the saturation concentration $C_{C, \text { sat }}$ representative of the $\mathrm{CO}_{2}$ solubility:

$$
C_{C} \triangleq \Pi C_{C, \text { sat }} \quad \Pi \in[0,1]
$$

The differential equation can then be written as

$$
C_{C, s a t} \frac{d \Pi}{d t}=C E R-\Pi D C_{C, s a t}-Q_{o u t}
$$

In agreement with (2) the carbon dioxide evolution rate $C E R\left[\mathrm{~L} \mathrm{CO}_{2} / \mathrm{L} \mathrm{h}\right]$ can be described by:

$$
C E R=\left[Y_{C / X} \mu+m_{C}+Y_{C / P} \pi\right] C_{X}
$$

under suitably controlled conditions for $\mathrm{pH}$. At any time during the fermentation, carbon dioxide arises from (i) growth and associated energy production (yield coefficient $Y_{C / X}\left[\mathrm{~L} \mathrm{CO}_{2} / \mathrm{g}\right.$ DW]), (ii) maintenance energy (specific rate $m_{C}$ $[\mathrm{L} \mathrm{CO} / \mathrm{g} \mathrm{DW} \mathrm{h}]$ ), and (iii) product biosynthesis and other possible specialized metabolism (yield coefficient $\left.Y_{C / P}\left[\mathrm{~L} \mathrm{CO}_{2} / \mathrm{g}\right]\right)$.

In most applications $C_{C, \text { sat }}$ is very low (i.e., $\mathrm{CO}_{2}$ solubility is very low), which means that carbon dioxide appears almost completely in gaseous form. Thus, letting $C_{C, s a t}=0$ is a meaningful singular perturbation. Equation (13) reduces to the following algebraic equation:

$$
Q_{\text {out }}=C E R
$$

which means that an on-line $\mathrm{CO}_{2}$ analysis of the effluent gas flow from the fermentor can be used to extract the variables needed. Note that $C_{C, \text { sat }}$ is not assumed to be equal to zero. It is only assumed that $C_{C, s a t}$ is small enough to neglect the terms $C_{C, s a t}(d \Pi / d t)$ and $\Pi D C_{C, s a t}$ in differential equation (13). Calam and Ismail (1980) reported the following slightly simplified relation in the case of penicillin $\mathrm{G}$ fermentation:

$$
C E R=Y_{C / X} \mu C_{X}+m_{C} C_{X}+k_{P}
$$

Based on experimental results, a constant value $k_{P}\left[\mathrm{~L} \mathrm{CO}_{2} / \mathrm{L} \mathrm{h}\right.$ representing the contribution of product synthesis is proposed, instead of a term involving the penicillin production rate. This can be motivated as follows. First, during the main production period the rate of biosynthesis is remarkably steady. Second, it is known that penicillin production is accompanied by decomposition (see, e.g., the hydrolysis constant $k_{h}$ in the penicillin model of Section 2.2). Therefore, it seems possible that as production later appears to slow down, biosynthesis itself may be continuing or may be diverted to non-antibiotic substances.

In the following algorithm the adaptive observer for $C_{X}$ and $\mu$ is inspired by (Di Massimo et al., 1989). This is only a partially adaptive observer, as some model constants are required a priori.

\section{Algorithm 3}

Step 1: Estimation of $\mu$ and $C_{X}$

$$
\begin{aligned}
\frac{d \hat{C}_{X}}{d t} & =\hat{\delta}-\hat{C}_{X} u / V+\omega(C E R-C \hat{E} R) \\
\frac{d \hat{\delta}}{d t} & =\gamma(C E R-C \hat{E} R) \\
C \hat{E} R & =Y_{C / X} \hat{\delta}+m_{C} \hat{C}_{X}+k_{P} \\
\hat{\mu} & =\frac{\hat{\delta}}{\hat{C}_{X}} \\
\hat{\sigma} & =\hat{\mu} / Y_{X / S}+m+\mathcal{C}
\end{aligned}
$$

Step 2: Calculation of the controller action $u$

$$
\begin{aligned}
u_{0} & =\frac{\hat{\sigma} \hat{C}_{X}-\lambda\left(\hat{\mu}-\mu^{*}\right)}{C_{S, \text { in }}} V \\
u & = \begin{cases}u_{0} & \text { if } 0 \leq u_{0} \leq U_{M A X} \\
0 & \text { if } u_{0} \leq 0 \\
U_{M A X} & \text { if } u_{0} \geq U_{M A X}\end{cases}
\end{aligned}
$$

\section{REMARKS}

1. The time-varying parameter $\hat{\delta}$ can be interpreted as an estimate of the biomass growth rate $\mu C_{X}[\mathrm{~g} \mathrm{DW} / \mathrm{L} \mathrm{h}]$. During estimation of $\sigma$ in Step 1 , the contribution of $\pi / Y_{P / S}$ is replaced by the constant term $\mathcal{C}[\mathrm{g} / \mathrm{g}$ DW $\mathrm{h}]$. This can be motivated as follows. During production, the objective is to keep the specific growth rate $\mu$ constant. In the case of $\mu$ and $\pi$ functions of $C_{S}$ only, this corresponds to keeping $C_{S}$-and thus also $\pi$ - constant during production. In any other case this is at least an excellent approximation. Observe that the expression for $\hat{\sigma}$ has now exactly the same form as model equation (14) for CER.

2. In the denominator of the controller action in Step 2 substrate concentration $C_{S}$ is considered negligible as compared with $C_{S, \text { in }}$.

3. Note that this scheme requires the a priori knowledge of the parameters $Y_{C / X}, m_{C}$, and $k_{P}$, and $Y_{X / S}, m$, and $\mathcal{C}$. In addition, if the endoge- 
nous fractions $f_{m}$ and $f_{P}$ are different from zero, their value should be known as well. Clearly, this is the price to pay for estimating state variables using only on-line measurements of easily accessible auxiliary variables.

4. However, this estimation procedure has an additional benefit over the algorithms presented in Sections 3 and 4 when scaling up the production from a pilot plant towards an industrial fermentor. When measuring substrate and/or biomass on-line, it becomes very important where to place the sampling devices on such a large reactor (e.g., actual penicillin production on an industrial scale takes place in fermentors of about $150000 \mathrm{~L}$ ). Due to an imperfectly mixed reactor, the question is whether a locally determined concentration is representative of the whole reactor contents. On the other hand, an analysis of the effluent gas from the fermentor provides in some sense averaged values of the reactor state which can be used immediately in the feed rate controller.

5. Obviously, this algorithm can also be implemented from time $t=0$ on by considering the setpoint for production $\mu^{*}$ as the reference from $t=0$ on. In case of a substrate inequality constraint during growth, the reference profile has the form shown in the lower plot of Fig. 3 with $C_{S, M A X}$ and $C_{S}^{*}$ replaced by the corresponding values for $\mu$.

6. As in Sections 3 and 4 , a continuous-time process model and a discrete-time version of the above estimator and controller have been used. Between sampling instants -at distance $\Delta T$ - the controller action $u_{k}$ is kept constant.

\subsection{Simulation results}

Some simulation results for the penicillin $\mathrm{G}$ model presented in Section 2.2 are shown in Fig. 6.

The model constants required in the estimator of $C E R$ are set equal to $Y_{C / X}=0.4\left[\mathrm{~L} \mathrm{CO}_{2} / \mathrm{g} \mathrm{DW}\right.$, $m_{C}=0.01\left[\mathrm{~L} \mathrm{CO}_{2} / \mathrm{g} \mathrm{DW} \mathrm{h}\right]$, and $k_{P}=0.3[\mathrm{~L}$ $\mathrm{CO}_{2} / \mathrm{L} \mathrm{h}$ ] (Nelligan and Calam, 1983). Using the parameters given in Table 2, the maximum value of $\pi$ is $\pi_{\max }=1.33310^{-3}[\mathrm{~g} / \mathrm{g} \mathrm{DW} \mathrm{h}$. Therefore, the constant $\mathcal{C}$ in the estimator of $\sigma$, which is an approximation of $\pi / Y_{P / S}$, is set equal to $\mathcal{C}=10^{-3}$ [g/g DW h].

The sampling interval $\Delta T$ is set equal to $\Delta T=0.1$ h. The initial substrate amount $S_{0}$ is set equal to $S_{0}=345 \mathrm{~g}$, which is optimal for heuristic $C_{S^{-}}$ control (Table 1). Tuning of the estimator, initialized with $\hat{C}_{x, k=0}=1.3[\mathrm{~g} \mathrm{DW} / \mathrm{L}], \hat{\delta}_{k=0}=0.13$ [g DW/L h], and $C \hat{E} R_{k=0}=0.375\left[\mathrm{~L} \mathrm{CO} \mathrm{CO}_{2} / \mathrm{L} \mathrm{h}\right]$, leads to the following efficient values: $\omega=5$, and $\gamma=25$. The controller parameter $\lambda$ is set equal to $\lambda=50$.
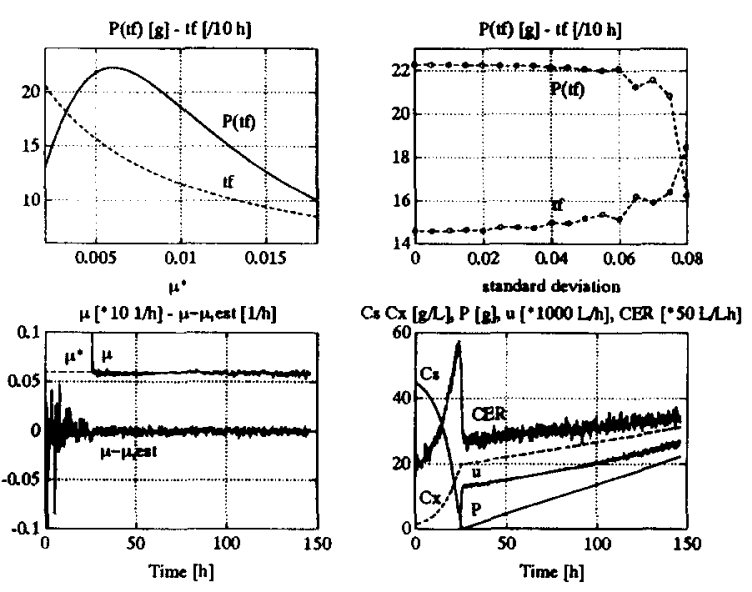

Fig. 6. Optimal adaptive control: on-line measurements of $C E R$. Upper left plot: optimization of $P\left(t_{f}\right)$ as function of $\mu^{*}$. Upper right plot: sensitivity of optimal values to increasing measurement noise. Lower plots: time profiles for the set-point $\mu^{*}=0.0061 / \mathrm{h}$

The upper left plot of Fig. 6 shows the optimization of the final product amount $P\left(t_{f}\right)$ with respect to the set-point $\mu^{*}$ for the specific growth rate during production, and the corresponding values of the final time $t_{f}$. The optimum occurs at $\mu^{*}=0.006[1 / \mathrm{h}]$. As in Section 4, a trade-off can be made between $P\left(t_{f}\right)$ and $t_{f}$ with respect to $\mu^{*}$. Furthermore, from the shape of the function $P\left(t_{f}\right)$ versus $\mu^{*}$ it can be concluded again that in practice three experiments should suffice to optimize the process.

The lower plots show the time profiles for this optimal set-point $\mu^{*}=0.006[1 / \mathrm{h}]$, while assuming a zero mean white noise on the measurements of $C E R$ with standard deviation std $(C E R)=0.025$ $\mathrm{L} \mathrm{CO}_{2} / \mathrm{L} \mathrm{h}$. This represents an admissible standard deviation of $5 \%$ for $C E R$ within the order of magnitude $C E R=\mathcal{O}[0.5] \mathrm{L} \mathrm{CO}_{2} / \mathrm{L} \mathrm{h}$ during production. The lower left plot illustrates the convergence of the estimator for the specific growth rate $\mu$, and the regulation of the actual specific growth rate $\mu(t)$ towards its set-point $\mu^{*}=0.006$ $1 / \mathrm{h}$. As in Sections 3 and 4 , the proposed algorithm has converged shortly after the beginning of the production phase. The right plot shows the noisy measurements of $C E R$, the adaptive control action $u_{k}$, together with the corresponding profiles for the state variables. The final product amount is $P\left(t_{f}\right)=22.273 \mathrm{~g}$ at $t_{f}=146.275 \mathrm{~h}$, which again comes very close to the optimal value of $P_{\text {opt }}\left(t_{f}\right)=22.606 \mathrm{~g}$ (Table 1).

The upper right plot illustrates the robustness of the optimal final product amount $P\left(t_{f}\right)$, and the corresponding final time $t_{f}$, with respect to increasing standard deviation of the measurement noise on the carbon dioxide evolution rate 
Table 3 Optimal adaptive control: a unifying approach

optimal control

$\downarrow$

heuristic control

$\downarrow$

optimal adaptive control model-sensitive, open-loop

microbiological/biochemical process knowledge

mathematical analysis of optimal control

model-independent control objective

linearizing control

adaptive state and parameter estimation

robust, nearly optimal performance
CER. It can be seen that the optimal values are more or less insensitive to this measurement noise, for standard deviations up to $\operatorname{std}(C E R)=0.06 \mathrm{~L}$ $\mathrm{CO}_{2} / \mathrm{L} \mathrm{h}$. For $C E R$ within the order of magnitude $C E R=\mathcal{O}[0.5] \mathrm{L} \mathrm{CO}_{2} / \mathrm{L} \mathrm{h}$ during production, this represents an admissible standard deviation of more than $10 \%$.

\section{CONCLUSIONS}

The main contribution of this paper was to present a unifying methodology for optimization of biotechnological processes, namely optimal adaptive control, by combining concepts and techniques from both optimal control and adaptive linearizing control. As an example, substrate feed rate controllers have been designed for a class of biotechnological processes, characterized by a decoupling between biomass growth and product formation.

It has been illustrated how the information obtained during preliminary optimal control studies leads to the design of easy-to-implement adaptive controllers. The optimal adaptive control procedure is summarized in a schematic way in Table 3 .

The design consists of the following steps.

Step 1 Derivation of the optimal control solution to the given optimization problem, under the assumption of a perfectly known process model.

Step 2 Derivation of nearly optimal heuristic controllers, based on a careful analysis of the optimal control solution of Step 1 from both the biochemical and the mathematical point of view. This second step itself consists of:

1. Detection of process variables which characterize the optimal control solution, such as a concentration, a specific rate, ...

2. Construction of a reference profile for the characteristic process variable as a function of time.
As such, the optimization problem of Step 1 is replaced by a more common tracking control problem, for which feedback control loops are designed in Step 3.

Step 3 Nonlinear adaptive implementation of the derived heuristic controller in two steps:

1. Embedding of the heuristic controller within a nonlinear linearizing controller.

2. Adaptive estimation of the states and parameters which are not available on-line. According to the minimum modeling principle, no assumption is made concerning the exact analytic nature of the specific rates needed in the control algorithm.

The optimal adaptive controllers derived in this way combine a nearly optimal performance with good robustness properties against modeling uncertainties and process disturbances.

To illustrate the method and the results obtained, simulation results have been given for the penicillin $G$ fed-batch fermentation process. Three possible implementations have been presented, depending on which variables are available by means of on-line measurements. The trade-off between on-line measurement requirements (such as accessibility and accuracy) and a priori information needs (such as yield and maintenance coefficients) has been clearly illustrated.

\section{ACKNOWLEDGMENTS}

This paper was presented in reduced form at the 5 th International Conference on Computer Applications in Fermentation Technology and 2nd IFAC Symposium on Modeling and Control of Biotechnical Processes, March 29-April 2, 1992, Keystone (Colorado). Author Jan F. Van Impe is a senior research assistant with the Belgian National Fund for Scientific Research (N.F.W.O.). This paper presents research results of the Belgian Programme on Interuniversity Attraction Poles initiated by the Belgian State, Prime Minister's Office, Science Policy Programming. The scientific responsibility rests with its authors. 


\section{NOMENCLATURE}

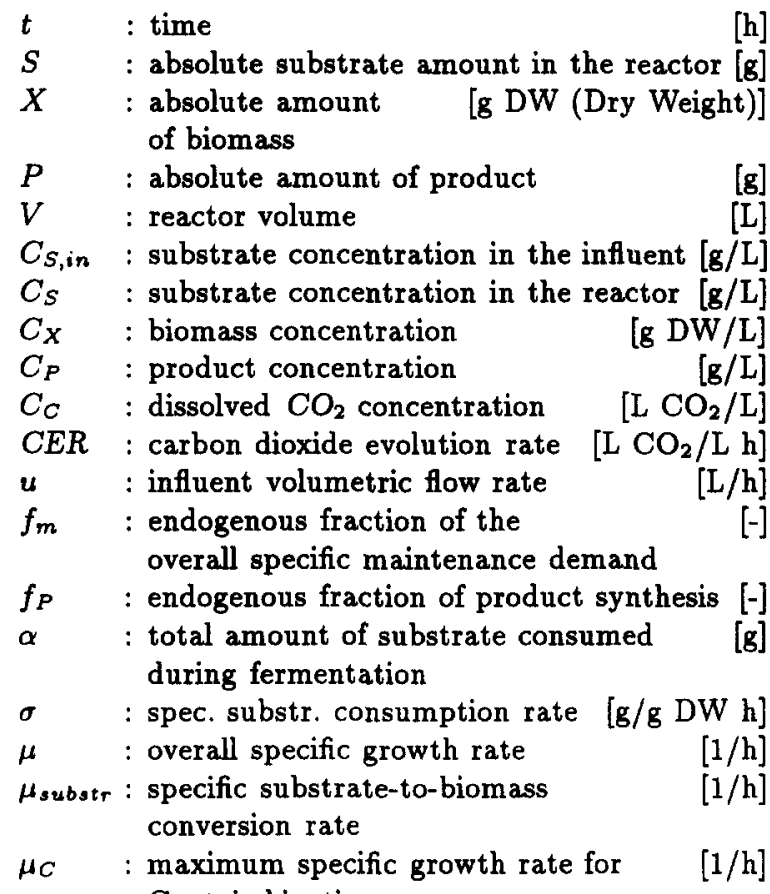
Contois kinetics

$K_{X} \quad$ : Contois saturation constant for [g/g DW] substrate limitation of biomass production

$\mu_{M} \quad$ : maximum specific growth rate for $[1 / \mathrm{h}]$ Monod kinetics

$K_{S} \quad$ : Monod saturation constant for substrate limitation of biomass production

$\pi \quad$ : specific production rate $\quad[\mathrm{g} / \mathrm{g} \mathrm{DW} \mathrm{h}]$

$\pi_{m} \quad$ : specific production constant [g/g DW h]

$K_{P} \quad$ : Monod saturation constant for $\quad[\mathrm{g} / \mathrm{L}]$ substrate limitation of product formation

$K_{I} \quad$ : substrate inhibition constant for $\quad[\mathrm{g} / \mathrm{L}]$ product formation

$m \quad$ : overall specific maintenance demand

$[\mathrm{g} / \mathrm{g} \mathrm{DW} \mathrm{h}]$

$m_{C} \quad$ : spec. $\mathrm{CO}_{2}$ production $\quad\left[\mathrm{L} \mathrm{CO}_{2} / \mathrm{g} \mathrm{DW} \mathrm{h]}\right.$ rate in maintenance processes

$k_{h} \quad:$ product degradation constant $\quad[1 / \mathrm{h}]$

$k_{P}: \mathrm{CO}_{2}$ due to production $\quad\left[\mathrm{L} \mathrm{CO}_{2} / \mathrm{L} \mathrm{h}\right]$

$Y_{X / S}$ : cell mass on substrate yield coeff. [g DW/g]

$Y_{P / S}:$ product on substrate yield coeff. $[\mathrm{g} / \mathrm{g}]$

$Y_{C / P}: \mathrm{CO}_{2}$ on product yield coefficient $\left[\mathrm{L} \mathrm{CO}_{2} / \mathrm{g}\right]$

$Y_{C / X}: \mathrm{CO}_{2}$ on biomass yield coeff. [L $\mathrm{CO}_{2} / \mathrm{g} \mathrm{DW}$ ]

\section{REFERENCES}

Bajpai, R.K. and M. Reuß (1980). A mechanistic model for penicillin production. J. Chem. Tech. Biotechnol., 30, 332-344

Bajpai, R.K. and M. Reuß (1981). Evaluation of feeding strategies in carbon-regulated secondary metabolite production through mathematical modelling. Biotechnol. Bioeng., 23, 717738
Bastin, G. and D. Dochain (1990). On-line estimation and adaptive control of bioreactors. Elsevier Science Publishing Co.

Bastin, G. and J.F. Van Impe (1995). Nonlinear and adaptive control in biotechnology: a tutorial. European Journal of Control, (submitted)

Calam, C.T. and B.A.-K. Ismail (1980). Investigation of factors in the optimisation of penicillin production. J. Chem. Tech. Biotechnol, 30, 249-262

Di Massimo, C., A.C.G. Saunders, A.J. Morris and G.A. Montague (1989). Nonlinear estimation and control of mycelial fermentations. Proceedings of the American Control Conference, June 21.23 1989, Pittsburgh (USA), 1994-1999

Goodwin, G.C. and K.S. Sin (1984). Adaptive filtering, prediction and control. Prentice-Hall, Englewood Cliffs, New Jersey

Kleman, G.L., J.C. Chalmers, G.W. Luli and W.R. Strohl (1991). A predictive and feedback control algorithm maintains a constant glucose concentration in fed-batch fermentations. Appl. Environ. Microbiol., 57, 910-917

Modak, J.M., H.C. Lim and Y.J. Tayeb (1986). General characteristics of optimal feed rate profiles for various fed-batch fermentation processes. Biotechnol. Bioeng., 28, 1396-1407

Nelligan, I. and C.T. Calam (1983). Optimal control of penicillin production using a mini-computer. Biotechnology Letters, 5, 561-566

Nicolaï, B.M., J.F. Van Impe, P.A. Vanrolleghem and J. Vandewalle (1991). A modified unstructured mathematical model for the penicillin $G$ fed-batch fermentation. Biotechnology Letters, 13 (7), 489-494

San, K.Y. and G. Stephanopoulos (1989). Optimization of fed-batch penicillin fermentation : a case of singular optimal control with state constraints. Biotechnol. Bioeng., 34, 72-78

Van Impe, J.F., B. Nicolai,, P. Vanrolleghem, J. Spriet, B. De Moor and J. Vandewalle (1992). Optimal control of the penicillin $G$ fed-batch fermentation: an analysis of a modified unstructured model. Chem. Eng. Comm., 117, 337-353

Van Impe, J.F., B. De Moor and J. Vandewalle (1993). Singular optimal control of fed-batch fermentation processes with growth/production decoupling and state inequality constraints. Preprints of the 12th World Congress International Federation of Automatic Control IFAC, $\underline{9}$, 125-128

Van Impe, J.F. (1994). Modeling and optimal adaptive control of biotechnological processes. Birkhäuser, Boston · Basel - Berlin (in preparation) 